\title{
A Combined Radial Basis Function Model for Fingerprint Distortion
}

\author{
Xuefeng Liang ${ }^{1}$, Tetsuo Asano ${ }^{1}$ and Hui Zhang ${ }^{2}$ \\ 1 Japan Advanced Institute of Science and Technology, 1-1, Asahidai, Nomi, Ishikawa \\ 923-1292, Japan \\ 2 Southwest University of Science and Technology, Mianyang, Sichuan 621010, China \\ \{xliang,t-asano\}@jaist.ac.jp, whgdzh@yahoo.com.cn
}

\begin{abstract}
Most fingerprint recognition techniques are based on minutiae matching and have been well studied. However, this technology still suffers from problems associated with the handling of poor quality impressions. One problem besetting fingerprint matching is distortion. Distortion changes both geometric position and orientation, and leads to difficulties in establishing a match among multiple impressions acquired from the same finger tip. In this paper, according to the particularity of fingerprint distortion, we propose a combined radial basis function (RBF) model, which separately builds rigid and nonrigid transformations, for attacking the distortion problem. Combined RBF model provides more accurate mapping function between a possible matched-pair. Experiments on real data demonstrate the efficacy of the proposed method in improving the compensation of fingerprint distortion.
\end{abstract}

\section{Introduction}

Automatic fingerprint identification systems (AFIS) are used for personal identification. AFIS are usually based on minutiae matching [6,15]. Minutiae, or Galton's characteristics [7] are local discontinuities in terms of ending and bifurcations of the ridge flow patterns that constitute a fingerprint. All current AFIS require the matching algorithms to become more tolerant with respect to some factors which prevent the false rejection rate (FRR) from decreasing beyond a certain limit. One of the main difficulties in matching two fingerprint samples of the same finger is to compensate non-linear distortions. Distortion arises from the elasticity of finger skin, the pressure and movement of fingers during image capture. It changes the spatial location and orientation of minutiae, and then leads to great difficulties in establishing a match among multiple images acquired from a single finger.

There have been several attempts to account for the elastic distortions in fingerprint images. Conventional matching techniques use tolerance boxes. In order to tolerate minutiae pairs that are apart because of distortion, and to decrease the FRR, the tolerance boxes can be enlarged. Jain et al. [8] and Luo, Tian and $\mathrm{Wu}[10]$ defined tolerance boxes in polar coordinates. In their methods, in order to compensate for the effect of distortion, the size of the tolerance 
boxes is incrementally increased moving from the center toward the borders of the fingerprint region. However, this still increased the probability of false acceptance rate (FAR). Senior and Boole [14] proposed a canonical model. Their model normalizes a fingerprint image to a canonical form in which all the ridges are equally spaced. This model can actually correct traction deformation very well, but torsion deformation cannot be adequately corrected. Cappelli, Maio and Maltoni [5] proposed a distortion model which defines three distinct distortion regions according to different pressure. Experiments showed that this model provides an accurate description of the elastic distortion. However, the parameters of this model should be given by experiments, not automatically. So, this model is not yet used in AFIS. Asker M. Bazen and Sabih H. Gerez [2] used a thin-plate spline (TPS) model to align all possible matched pair of impressions. Owing to iteratively aligning minutiae between input and template impressions, a risk of forcing an alignment between impressions originating from two different fingers arises, and leads to a higher FAR. Arun Ross, Sarat C. Dass and Anil K. Jain [12] attempted to build an average deformation model from multiple impressions of the same finger using a TPS model. Since we cannot guarantee that any given pre-captured impression contains all kinds of distortions, this average deformation model does not have sufficient competence for matching.

In this paper, we propose a combined RBF model to compensate fingerprint elastic distortion. The main contributions of this work is that proposed combined RBF model separately builds rigid and nonrigid transformations based on the particularity of fingerprint distortion, and then provides more accurate mapping function between a possible matched-pair. Experiments on FVC 2000 Database and scanned fingerprints with heavy distortion show our combined RBF model has a higher ability to compensate distortion.

The rest of this paper is organized as follows: In Section 2, we roughly introduce fingerprint deformation and review the basic theory of RBF interpolation. Section 3 describes our combined RBF model for fingerprint distortion. Section 4 evaluates the proposed combined RBF model on two fingerprint databases. Finally, we present our conclusions in Section 5 .

\section{Fingerprint Distortion and Normal RBF}

The most conversional fingerprint matching techniques simply use rigid-Affine transformation to compensate distortion. However, they invariably lead to unsatisfactory matching results since the soft tissue of finger causes the distortion is basically elastic in nature. Actually, distortion is a combination of rigid and nonrigid transformations. Below, we briefly discuss the rigid-affine model and the nonrigid model.

\section{$2.1 \quad$ Rigid-Affine Model}

A spatial transformation is considered rigid if the spatial distance between any consecutive points is preserved. Rigid transformation can be decomposed into 
a translation and/or a rotation. A translation is a constant displacement over space. A rigid model is a constrained subset of an affine model. So, an affine transformation can be decomposed into a linear transformation and a translation. In $2 \mathrm{D}$, a simple affine transformation can be expressed by a linear polynomial of the following equation:

$$
f_{k}(\vec{x})=a_{1 k}+a_{2 k} x+a_{3 k} y \quad k=1,2
$$

where $\vec{x}=(x, y)$.

With a rigid-affine model, fingerprint structures retain their shape and form during matching. This limits their practical application. To address the issue of deformable behavior, we need an elastic model in which a structure may not necessarily retain its shape or form during transformation.

\subsection{Nonrigid Model}

A nonrigid transformation is opposite to a rigid one, in which the spatial distance between any consecutive points can not be preserved owing to various pressures on finger surface. In this case, finger surfaces are compressed on themselves or stretched. Such behavior is synonymous with the characteristics of a higher degree polynomial:

$$
f_{k}(\vec{x})=\sum_{i=0}^{n}\left(a_{i k} x^{i}+b_{i k} y^{i}\right) \quad k=1,2 ; n \geq 3
$$

\section{$2.3 \quad$ A Normal RBF Model}

As property of finger tips, there is an observation: even if an elastic distortion is applied to a fingerprint image, every minutiae always keeps the same neighbor structure. Several papers use this idea to design local matching algorithms $[3,4$, 11]. However, such algorithms are not sufficient for fingerprint matching because only local information is used rather than a whole image. But, this idea give us a hint that, local similarity can help AFIS to select the possible matched candidates.

With local similarities among possible matched fingerprints, approximating a non-linear mapping function between two impressions is a typical interpolation problem. Scattered data interpolation is fundamental in deriving a smooth spatial transformation from the correspondence of minutiae between a pair of impressions, namely control-points. The advantage of using scattered data interpolation methods to model deformation is that they need fewer control-points, or the control-points are sparsely distributed. Control-points in input and template impressions can be extracted by an indexing algorithm using Delaunay triangulation [9]. One way of approaching interpolation is to use Radial Basis Function (RBF). RBF offers several advantages: first of all, the geometry of the control-points is by no means restricted, this implies that the distribution of 
control-points can be both sparse and irregular; secondly, the RBF provides easily controllable behavior that can be tailored to meet specific requirements. An RBF may be purely deformable, or it may contain some form of linear component, allowing both local and global deformations.

Generally, a RBF spatial transformation in $d$ dimensions, denoted $T(\vec{x})$, is composed of $k$ mapping functions $k=1, \ldots, d$ such that:

$$
T(\vec{x})=\left[f_{1}(\vec{x}), \ldots, f_{k}(\vec{x}), \ldots, f_{d}(\vec{x})\right]
$$

where $f_{1}(\vec{x})$ represents the mapping function in the first dimension etc. Each of the mapping functions can be decomposed into a global component and a local component. Although the two components are distinct, they are evaluated almost simultaneously, giving rise to a single transformation. This decomposition enables a family of transformations to be defined where, if desired, the influence of each control-point can be controlled. Given $n$ corresponding control-point pairs, each of the $k$ mapping functions of the RBF has the following general form:

$$
f_{k}(\vec{x})=P_{m k}(\vec{x})+\sum_{i=1}^{n} A_{i k} g\left(r_{i}\right)
$$

The first component $P_{m k}(\vec{x})$ is the global linear transformation denoted by a polynomial of degree $m$. In 2 dimensions, the general form of a linear polynomial (see formula $1, m=1$ ) is used, making the global component an affine transformation. The latter component is the sum of a weighted elastic or nonlinear basis function $g\left(r_{i}\right)$, where $r_{i}$ denotes the Euclidean norm, such that:

$$
r_{i}=\left[\vec{x}-\vec{x}_{i}\right]^{\frac{1}{2}} \text { or } r_{i}=\left\|\vec{x}-\vec{x}_{i}\right\|
$$

The coefficients of the function $f_{k}(\vec{x})$ are determined by following conditions:

$$
f_{1}\left(\vec{x}_{j}\right)=u_{j} \quad \text { and } \quad f_{2}\left(\vec{x}_{j}\right)=v_{j} \quad \text { for } j=1, \ldots, n
$$

giving $n$ linear equations together with the additional compatibility constraints:

$$
\sum_{i=1}^{n} A_{i k}=\sum_{i=1}^{n} A_{i k} x_{i}=\sum_{i=1}^{n} A_{i k} y_{i}=0
$$

These conditions guarantee that the RBF is affine reducible. The coefficients of the basis function and the polynomial can now be found by solving the linear system:

$$
W=L^{-1} Y
$$

where

$$
W^{T}=\left[\begin{array}{lllllll}
A_{11} & A_{21} & \cdots & A_{n 1} & a_{11} & a_{21} & a_{31} \\
A_{12} & A_{22} & \cdots & A_{n 2} & a_{12} & a_{22} & a_{32}
\end{array}\right]
$$




$$
\begin{aligned}
& Y^{T}=\left[\begin{array}{lllllll}
u_{1} & u_{2} & \cdots & u_{n} & 0 & 0 & 0 \\
v_{1} & v_{2} & \cdots & v_{n} & 0 & 0 & 0
\end{array}\right] \\
& L=\left[\begin{array}{cc}
G & P \\
P^{T} & 0
\end{array}\right] \\
& G=\left[\begin{array}{cccc}
g\left(r_{11}\right) & g\left(r_{12}\right) & \cdots & g\left(r_{1 n}\right) \\
g\left(r_{21}\right) & g\left(r_{22}\right) & \cdots & g\left(r_{2 n}\right) \\
\vdots & \vdots & \ddots & \vdots \\
g\left(r_{n 1}\right) & g\left(r_{n 2}\right) & \cdots & g\left(r_{n n}\right)
\end{array}\right] \\
& P^{T}=\left[\begin{array}{cccc}
1 & 1 & \cdots & 1 \\
x_{1} & x_{2} & \cdots & x_{n} \\
y_{1} & y_{2} & \cdots & y_{n}
\end{array}\right]
\end{aligned}
$$

\section{A Combined RBF Model}

When the finger tip is orthogonally pressed against the plain surface of a fingerprint sensor, due to the convex surface and soft tissue of the finger, not all regions are orthogonal to the sensor surface. Therefore, the pressures on the fingerprint are not uniform, but monotonically decrease from the center (see Figure 1). We roughly separate a fingerprint image scanned by planar sensor into two regions [5]:

I rigid region. This is the closest contact region with the highest pressure, which normally does not allow skin slippage. In our method, the radii of region $\mathbf{I}$ is $1 / 3$ of radius of whole fingerprint region. In practice, consider 8 radials staring from the center of fingerprint region $O$ with orientation $0^{0}, \pm 45^{0}, \pm 90^{0}, \pm 135^{0}, 180^{0}$ with regard to $x$-axis respectively. Each of them intersects the external boundary of fingerprint region at $P_{i}, i=1, \ldots, 8$. Choosing a point $V_{i}$ on $\overrightarrow{O P}_{i}$ lets $\left|O P_{i}\right|=3\left|O V_{i}\right|$, and then orderly connect vertexes $V_{i}$ in clockwise direction. Let this octagon as the rigid region.

II nonrigid region. Pressure monotonically decreases from the boundary of region I to the external boundary of region II. The main elastic distortion is located in this region.

With this observation, we are ready to modify the method in previous section. From section 2.3, we know RBF consists of a linear transformation and a nonlinear transformation (see equation 4). All coefficients of both components are calculated simultaneously in the previous RBF model. Therefore, the controlpoints in nonrigid region II must affect the accuracy of the linear polynomial. 


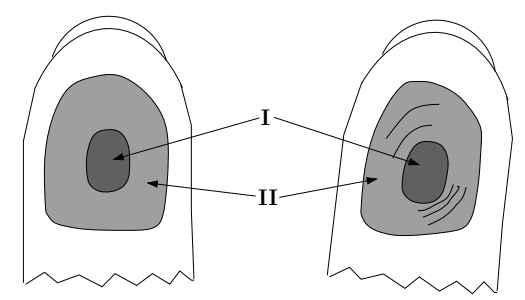

Fig. 1. Bottom views of a finger without and with distortion, two pressure touching regions.

To compensate fingerprint distortion, it is advantageous to more precisely compute the linear polynomial. According to the definition of rigid region $\mathbf{I}$, the minutiae in this region are almost relatively motionless, whether the impression contains distortion or not. Hence the control-points in region $\mathbf{I}$ can contribute to the polynomial of the linear transformation of the whole impression. The distortion in nonrigid region II is caused by the same linear transformation and the local nonlinear transformation synchronously. Our strategy is as follows: first, estimate the linear transformation $\left(P_{m k}(\vec{x})\right)$ of the whole impression by the control-points in region $\mathbf{I}$; second, remove the linear transformation effect of the control-points in region II, and then construct a purely deformable RBF model for region II $\left(f_{k}(\vec{x})-P_{m k}(\vec{x})=\sum_{i=1}^{n} A_{i k} g\left(r_{i}\right)\right)$; finally, compensate distortions in regions $\mathbf{I}$ and $\mathbf{I I}$, respectively. According to this idea, we modified the method and equations 8, 9, 10 as follows:

$$
W^{\prime}=L^{-1} Y^{\prime}
$$

where

$$
\begin{aligned}
& W^{\prime T}=\left[\begin{array}{lllllll}
A_{11} & A_{21} & \cdots & A_{n 1} & 0 & 0 & 0 \\
A_{12} & A_{22} & \cdots & A_{n 2} & 0 & 0 & 0
\end{array}\right] \\
& Y^{\prime T}=\left[\begin{array}{llllll}
u_{1}-\varphi_{11} & \cdots & u_{n}-\varphi_{n 1} & 0 & 0 & 0 \\
v_{1}-\varphi_{12} & \cdots & v_{n}-\varphi_{n 2} & 0 & 0 & 0
\end{array}\right] \\
& \varphi_{i k}=a_{1 k}+a_{2 k} x_{i}+a_{3 k} y_{i}
\end{aligned}
$$

Input: $\quad$ Minutiae sets $X$ and $U$ from input and template impressions respectively.

Output: Minutiae set $X^{\prime}$ after compensation by combined RBF model. 


\section{Build the combined RBF model.}

1. Substitute at least three paired control-points $\vec{x}_{i}^{c} \in X_{I}$ and $\vec{u}_{i}^{c} \in U_{I}$, which locate in rigid regions $\mathbf{I}$, into equation 1 , and then obtain $a_{i k}$.

2. $\forall \vec{x}_{i}^{c} \in X_{I I}$ and $\forall \vec{u}_{i}^{c} \in U_{I I}$, which locate in nonrigid regions II, Orderly compute equations 17, 16 and 14. Obtain $A_{i k}$.

\section{Compensate fingerprint distortion.}

3. For all non-control-points $\vec{x}_{i} \in X$

If $\vec{x}_{i} \in X_{I}$; Then $\vec{x}_{i}^{\prime} \leftarrow$ equation 1 .

End

If $\vec{x}_{i} \in X_{I I}$; Then $\vec{x}_{i}^{\prime} \leftarrow$ equation 4 .

To compensate distortion as good as possible, an appropriate basis function is necessary. A number of candidate 2D basis functions of RBF are given in Table 1. The choice of a basis function is determined by the interpolation conditions and the desired properties of the interpolation. Radial basis functions can be tailored to many specific needs, and their range of influence can be controlled by adjusting the parameters of the RBF.

Table 1. Basis Functions

\begin{tabular}{|l|c|c|}
\hline Basis Function & $g\left(r_{i}\right)$ & Parameters \\
\hline \hline Thin-Plate Spline(TPS) & $r_{i}^{2} \log r_{i}$ & - \\
\hline Wendland & $\left(1-r_{i}\right)_{+}^{4}\left(4 r_{i}+1\right)$ & - \\
\hline Multiquadric(MQ) & $\left(r_{i}^{2}+\delta\right)^{+\mu}$ & $\delta>0,0<\mu$ \\
\hline Inverse Multiquadric & $\left(r_{i}^{2}+\delta\right)^{-\mu}$ & $\delta>0,0<\mu$ \\
\hline Gaussian & $e^{\left(-r_{i}^{2} / \delta\right)}$ & $\delta>0$ \\
\hline
\end{tabular}

As Table 1 illustrates, both the TPS and multiquadric basis functions monotonically increase with distance from the center. In contrast, the Wendland, Gaussian and Inverse MQ basis functions monotonically decrease with distance from the center. Since fingerprint distortion is elastic rather than viscous (like brain tissue for example), the influence of control-points on the compensated results increases with distance at a certain range from the center. Therefore, MQ and TPS suit elastic deformation. Moreover, fingerprint distortion is not globally uniform. For instance, if one side of a traction deformation center is compressed, the opposite side must be dilated. We would like to control the area of influence of basis function. Locality parameters can perform this task. They give less weight to distant control-points and more weight to neighboring ones. Locality parameter uses unique values $\delta_{i}$, which is the distance to the nearest neighboring control-point,

\footnotetext{
${ }^{3}$ Formula 1 shows that there are six coefficients to be specified. This means at least three paired control-points in region $\mathbf{I}$ are required. Delaunay triangle alignment algorithm in [9] preforms this task. Experiments show that this demand can be easily filled.
} 
for each control-point. This essentially allows the distortion to be softer where control-points are widely spaced and stronger where they are closer together. This more effectively works on fingerprint minutiae. Using this adaptive locality parameter, MQ is modified as:

$$
g\left(r_{i}\right)=\left(r_{i}^{2}+\delta_{i}\right)^{\mu}
$$

where $\delta_{i}=\min _{i \neq j}\left(r_{i j}\right)(j=1, \ldots, n)$.

As TPS has no parameters, clearly it cannot adapt itself to a particular deformation problem.

After distortion compensation, our minutiae matching conditions is: for the corresponding minutiae in both sets, if the differing in location and orientation less than a threshold, they are defined as matched. The matching score $\mathbf{S}$ is calculated by

$$
\mathrm{S}=\frac{n_{\text {match }}^{2}}{n_{1} n_{2}}
$$

where $n_{\text {match }}$ is the number of matched minutiae, $n_{1}$ and $n_{2}$ are the number of minutiae in the input and template impressions, respectively.

\section{Experiments and Comparisons}

To show how the combined RBF model can compensate fingerprint distortion, two numerical evaluation measures are employed.

1. An average distance of all minutiae pairs between the compensated input impression and the template impression:

$$
\bar{r}=\frac{\sum_{i=1}^{n}\left[\left(u_{i}^{\prime}-u_{i}\right)^{2}+\left(v_{i}^{\prime}-v_{i}\right)^{2}\right]^{\frac{1}{2}}}{n}
$$

where $f_{1}\left(\vec{x}_{i}\right)=u_{i}^{\prime}$ and $f_{2}\left(\vec{x}_{i}\right)=v_{i}^{\prime}$ for $i=1, \ldots, n$, and point $\left(x_{i}, y_{i}\right)$ is a minutia in input impression, similarly, point $\left(u_{i}, v_{i}\right)$ is the corresponding minutia in template impression. $n$ is the number of the corresponding minutiae pairs after compensation.

2. Matching performance (ROC) curves plotting the false rejection rate (FRR) against the false acceptance rate (FAR) at various thresholds.

We evaluated our method by testing it on Database FVC2000 which consists of 880 fingerprints, 8 impressions each of 110 distinct fingers. In addition, we also scanned 80 fingerprints with heavy distortion from a FUJITSU Fingerprint Sensor (model: FS-210u), 4 impressions each of 20 distinct fingers. Size and resolution are $300 \times 300$ and 500dpi respectively.

As scanned fingerprints are heavily distorted, the first evaluation measure (average distance) is only applied on them. We list results of a normal RBF model with TPS basis function, proposed combined RBF model and a rigid 
transformation in Table 2 for an easy comparison. Results show combined RBF model approximates a best nonlinear transformation. A visual result is shown in Figure 2. (a) and (b) are impressions without and with distortion. (c), (d) and (e) are compensation results using a normal RBF model with TPS basis function, the proposed combined RBF model and a rigid transformation, respectively. Circles in these figures denote their own minutiae; crosses denote the mapped minutiae from impression with distortion. (d) clearly shows that most mapped minutiae from input impression are very close to the minutiae to be matched in template impression.

Table 2. Evaluation of various basis functions

\begin{tabular}{|l|c|c|}
\hline Method & $\bar{r}$ & Parameters \\
\hline \hline Normal RBF(TPS) model & 8.5643 & - \\
\hline Combined RBF model & 5.2136 & $a_{i k}, \delta_{i}, \mu^{*}=0.2$ \\
\hline Rigid Transformation & \multicolumn{2}{|l|}{$\bar{r}_{\text {rigid }}=17.5852$} \\
\hline
\end{tabular}

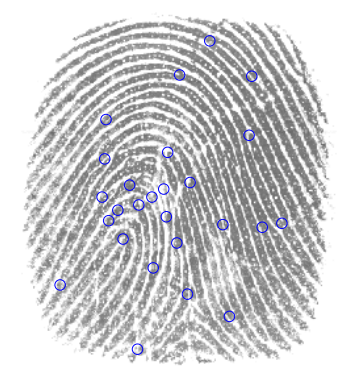

(a)Regular impression.

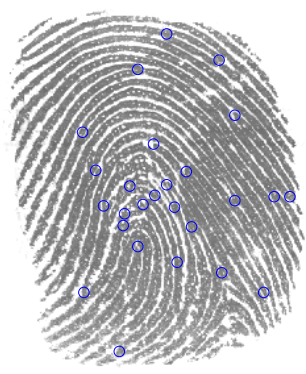

(b)Impression with distortion.

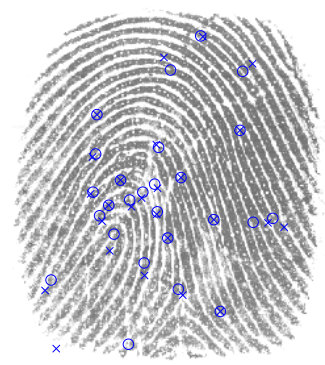

(c)Normal RBF(TPS) model.

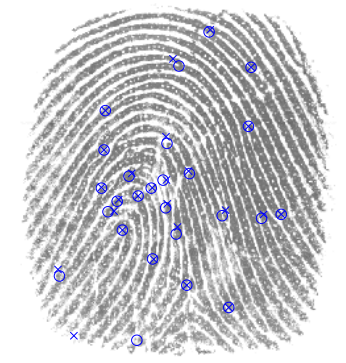

(d)Combined RBF model.

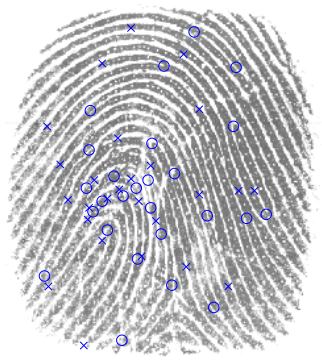

(e)Rigid transformation.

Fig. 2. Minutiae correspondence before and after distortion compensation. 
The matching performance was evaluated on both FVC2000 Database and scanned fingerprints. Due to the lack of a benchmark of minutiae matching performance, we compared our matching method with the TPS-based algorithm in [2] on two aspects: computing time and accuracy of identification. For these two methods, a same tolerance box with radius $r=5$ was used. Since TPSbased algorithm iteratively registers minutiae between two fingerprints, they are time consuming and lead to a higher risk of matching two similar fingerprints coming from distinct fingers. On the contrary, our method uses a more precise combined RBF model and parameters tailor the MQ basis function more suitable for elastic distortion. So it does not need iteration of registering minutiae. Our matching method costs $30 \mathrm{~ms}$ for one matching rather than $107 \mathrm{~ms}$ of TPS-based algorithm on average, so computing time is around two times faster. Matching performance (ROC) curves are plotted in Figure 3. For the data in FVC2000, our method is better than TPS-based algorithms, but improvement is not so much; for the scanned fingerprints with heavy distortion, our method is obviously more accurate. We believe that this is due to combined RBF model more precisely describes fingerprint distortion and the MQ basis function can be tailored by parameters for this particular problem. To sum up, the combined RBF model not only provides computational efficiency, but also leads to better performance in matching.

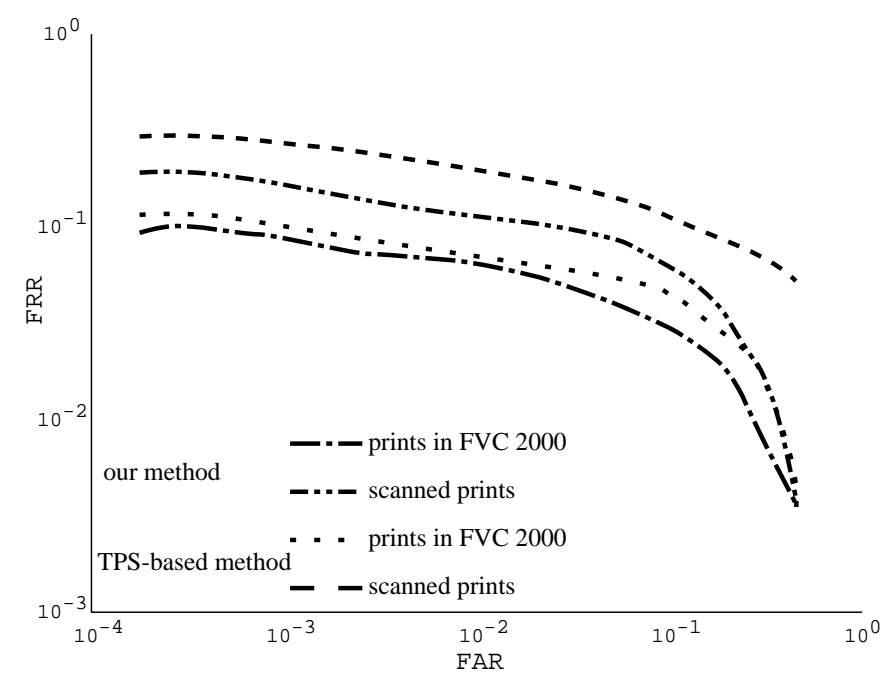

Fig. 3. ROC curves on FVC2000 DB and scanned fingerprints obtained with the proposed method and the algorithm in [2].

However, if two fingerprint images from a same finger have less than $60 \%$ common fingerprint region, in other words, rigid regions on two impressions 
have no common region, our combined RBF model will become a normal RBF model.

\section{Discussion and conclusion}

This paper introduces a combined RBF model to compensate fingerprint elastic distortion. According to the particularity of fingerprint distortion, we proposed a combined RBF model, which separately builds rigid and nonrigid transformations, for attacking the distortion problem. Combined RBF model provides more accurate mapping function between a possible matched minutiae pair. Experiments show our combined RBF model has a better matching performance compared with [2].

Our future efforts will target a more in-depth study of the properties of fingerprint distortion and the design of an effective and efficient optimization basis function.

\section{Acknowledgment}

Research for X.F. Liang was conducted as a program for the "Fostering Talent in Emergent Research Fields" in Special Coordination Funds for Promoting Science and Technology by Ministry of Education, Culture, Sports, Science and Technology. Research for T. Asano was partially supported by the same Ministry, Grant-in-Aid for Scientific Research (B) and Exploratory Research. FUJITSU LABORATORIES LTD. was extremely helpful in providing devices for our research.

\section{References}

1. Arad, N., Reisfeld, D.: Image warping using few anchor points and radial functions. Computer Graphics Forum. 14(1) (1995) 35-46.

2. Bazen, A. M., Gerez, S. H.: Elastic mniutiae matching by means of thin-plate spline models. Pattern Recognition. 36(8) (2003) 1859-1867.

3. Bebis, G., Deaconu, T., Georgiopoulos, M.: Fingerprint identification using delaunay triangulation. (1999) 452-459.

4. Bhanu, B., Tan, X. J.: Fingerprint indexing based on novel features of minutiae triplets. IEEE Trans. on PAMI. 25(5) (2003) 616-622.

5. Cappelli, R., Maio, D., Maltoni, D.: Modelling plastic distortion in fingerprint images. Proc. of CAPR. (2001) 369-376.

6. Farina, A., Kovács-Vajna, Z. M., Leone, A.: Fingerprint minutiae extraction from skeletonized binary images. Pattern Recognition. 32 (1999) 877-889.

7. Galton, F.: Fingerprints. London: Macmillan (1892).

8. Jain, A. K., Hong, L., Bolle, R. M.: On-line fingerprint verification. IEEE Trans. on PAMI. 19(4) (1997) 302-313.

9. Liang, X. F.: Fingerprint image analysis using computational geometric techniques. Ph.D. thesis. Japan Advanced Institute of Science and Technology. (2005) 68-72. 
10. Luo, X., Tian, J., Wu, Y.: A minutia matching algorithm in fingerprint verification. Proc. of ICPR. 4 (2000) 833-836.

11. Parziale, G., Niel, A.: A Fingerprint matching using minutiae triangulation. Proc. of ICBA 2 (2004) 241-248.

12. Ross, A., Dass, S. C., Jain, A. K.: Estimation fingerprint deformation. Proc. of ICBA. 2 (2004) 249-255.

13. Ruprecht, D., Müller, H.: Image warping with scattered data interpolation. IEEE Computer Graphics and Applications. 15(2) (1995) 37-43.

14. Senior, A., Bolle, R.: Improved fingerprint matching by distortion removal. IEICE Trans. on INF. \& SYST. E84-D(7) (2001) 825-832.

15. Kovács-Vajna, Z. M.: A fingerprint verification system based on triangular matching and dynamic time warping. IEEE Trans. on PAMI. 22(11) (2000) 1266-1276. 